\section{Occupational Exposure to Zoonotic Tuberculosis Caused by Mycobacterium caprae, Northern Greece, 2019}

\author{
Dimitrios Papaventsis, George Dougas, \\ Ourania Kalkouni, Simona Karabela, \\ Katerina Manika
}

\begin{abstract}
Author affiliations: Sotiria Chest Diseases Hospital, Athens, Greece (D. Papaventsis, S. Karabela); National Public Health Organization, Athens (G. Dougas, O. Kalkouni); G. Papanikolaou Hospital, Thessaloniki, Greece (K. Manika)
\end{abstract}

DOI: https://doi.org/10.3201/eid2707.204399

Pulmonary tuberculosis caused by Mycobacterium caprae was diagnosed in a 65-year-old goat breeder from northern Greece. This case represents a documented occupational transmission of $M$. caprae and highlights the importance of enhanced laboratory screening and increased surveillance for zoonotic tuberculosis control.

$M$ ycobacterium caprae, a member of Mycobacterium tuberculosis complex (MTBC), causes caprine tuberculosis (TB) (1). M. caprae has been isolated mainly in continental Europe and infects animals and humans (2-4). Although clinically indistinguishable from $M$. tuberculosis or M. bovis, $M$. caprae causes nearly one third of $M$. bovis-associated TB cases (5). Bovine TB control and pasteurization have made zoonotic TB rare; however, occupational risk exists for livestock farmers, veterinarians, slaughterhouse workers, butchers, and other persons working in close contact with livestock.

In August 2019, a 65-year-old male goat breeder from northern Greece was admitted to the Aristotle University Pulmonology Department at G. Papanikolaou Hospital (Thessaloniki, Greece) for weight loss, productive cough, and fever $<38.5^{\circ} \mathrm{C}$ that was not responding to a 10-day regimen of cefuroxime. He was a frequent smoker, reported chronic alcohol abuse, and had no other medical history. He reported living in the goat shelter, occasionally consuming unpasteurized milk, and having no contact with or hunting practices involving other animal species. Laboratory tests showed normocytic anemia (hemoglobin 10.4 $\mathrm{g} / \mathrm{dL}$ ) and an erythrocyte sedimentation rate of 112 $\mathrm{mm} / \mathrm{h}$. Chest radiograph and computed tomography scans revealed upper lobe cavities and infiltrations, predominantly on the left side (Figure, panels A-C). A tuberculin skin test result was $12 \mathrm{~mm}$ in size. TB was suspected; sputum samples were sent for molecular and bacteriologic testing to the National Reference Laboratory for Mycobacteria at Sotiria Chest Diseases Hospital (Athens, Greece). Acid-fast stain results were positive and MTBC detected by using TRCReady- 80 (Tosoh Corp., https://www.tosoh.com). We determined drug sensitivity on a strain grown on sodium pyruvate-LJ medium by Genotype MTBDRplus (Hain LifeScience, https:/ /www.hain-lifescience.de) and on solid media. We documented sensitivity to first-line anti-TB drugs and pyrazinamide. Because of the patient's occupation, laboratory investigation included zoonotic species. GenoType MTBC differentiated the isolate as $M$. bovis subspecies caprae.

We used the optimized 24-loci Genoscreen MIRUVNTR (mycobacterial interspersed repetitive unit-variable-number tandem-repeat) Typing Kit (Genoscreen, https://www.genoscreen.fr) on crude DNA, using 6 quadruplex PCR and fluorescent primers with capillary electrophoresis. Identification was performed with the MIRU-VNTRplus database (6). Because no match was detected after initial best-match analysis, we used a treebased identification scheme by applying the UPGMA method and using the database reference strains. Identification was confirmed with a unique MIRU-VNTR pattern (M.caprae_Kilkis:255326322553434243231432) showing close phylogenetic match with $M$. caprae reference strains from central and eastern Europe and clear genetic distance from the Iberian cluster, mainly characterized by absence of spoligotype spacers 30-33 (3) (Appendix Figure, https://wwwnc.cdc.gov/EID/ article/27/7/20-4399-App1.pdf).

The patient received a standard anti-TB regimen for 9 months and responded favorably. Cultures and acid-fast bacilli turned negative 10 days after treatment and were negative 2.5 months after treatment. Erythrocyte sedimentation rate dropped to $25 \mathrm{~mm} / \mathrm{h}$, and the patient gained $17 \mathrm{~kg}$. Chest radiograph and computed tomography scans showed remarkable improvement (Figure, panels D-F). Contact tracing did not reveal further human cases. Veterinary investigation identified no tuberculin skin test reactors among $>300$ goats of the epidemiologically linked herd.

Zoonotic TB causes an estimated 147,000 new human cases and 12,500 deaths annually worldwide (7). Zoonotic TB is a reemerging and underrecognized infection in Europe; 170 confirmed human cases were reported in 2018 (0.05 cases/100,000 population) (4). Among countries not officially TB-free, Greece, Ireland, and Spain reported a plateaued prevalence of $2 \%-5 \%$ in cattle herds over the past decade (4). The actual global burden of $M$. caprae disease is further underestimated because of differences in laboratory 

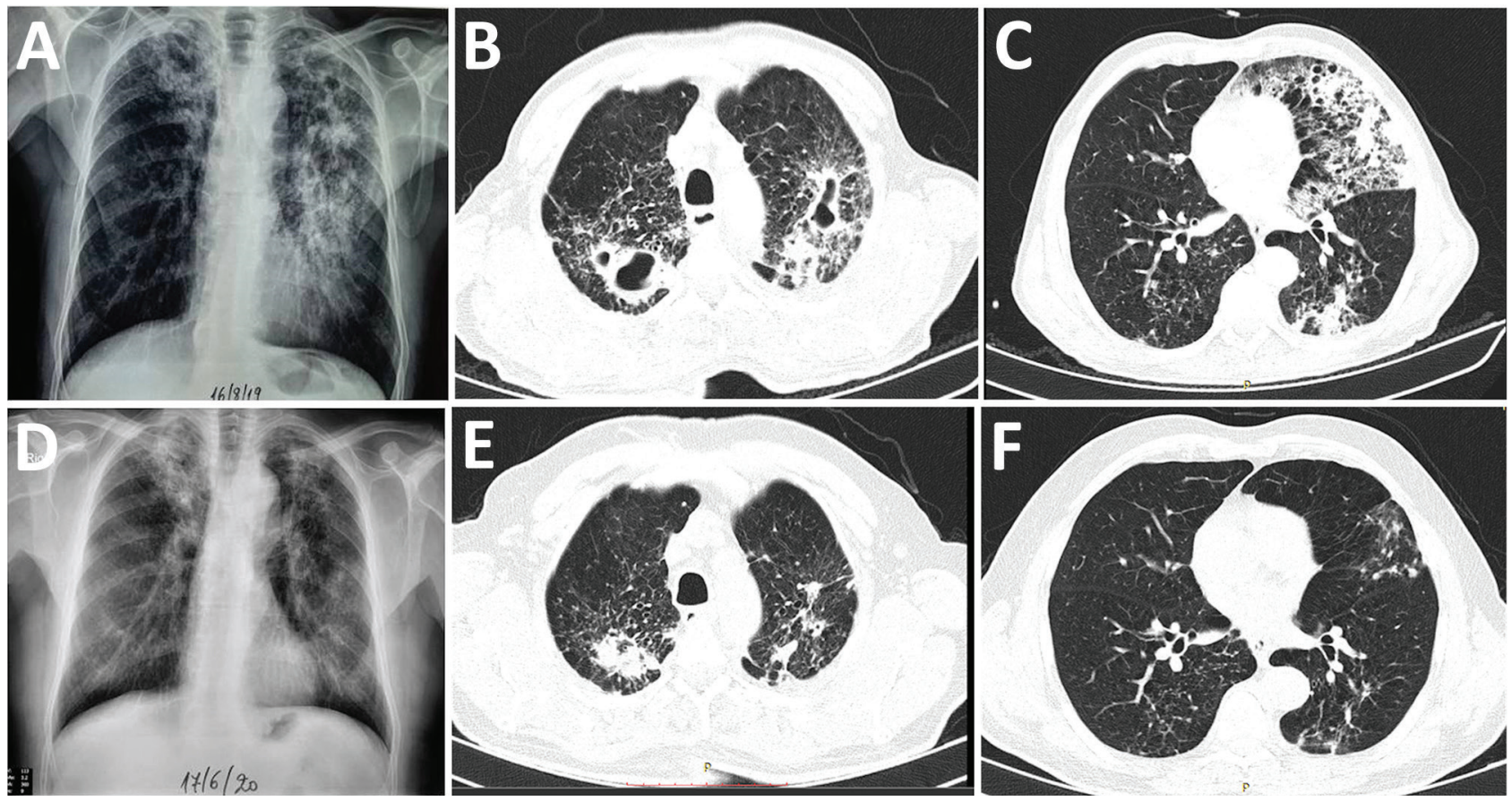

Figure. Comparison of chest radiographs and computed tomography scans before and after the end of treatment for a 65-year-old male goat breeder infected with Mycobacterium caprae, northern Greece, 2019. Multiple cavity infiltrations and opacities are shown on the chest radiographs $(A)$ and the computed tomography scan $(B, C)$, mainly in the left upper lobe. After treatment, significant improvement is shown by cavity closure and recession of opacities and infiltration on the chest radiograph (D) and on the chest computed tomography scans $(E, F)$.

capacity and lack of routine surveillance data. Zoonotic TB is a public health hazard, resulting in serious economic losses and having a substantial effect on poor and marginalized communities (7).

Contaminated food and airborne transmission pose considerable risks to persons in contact with infected animals or animal products (7). In this study, caprine TB was occupationally acquired by a goat farmer with a history of routine close contact with livestock. Pyrazinamide sensitivity and lung localization could have caused an initial misdiagnosis of $M$. tuberculosis infection. Since 2019, enhanced laboratory screening and surveillance for high-risk patients related to animal breeding have been used in Greece. Timely application of bacteriologic and genotyping techniques, less frequently used in routine laboratory investigation until recently, highlighted the role of $M$. caprae as a human pathogen. MIRU-VNTR revealed a unique isolate similar to patterns reported in animals in Eastern Europe and the Balkans $(3,8)$. Data on $M$. caprae zoonotic TB in Greece are limited. A dairy goat farm outbreak in northern Greece was the first documented in 2005; M. caprae caused pneumonia among animals, and the mortality rate reached $92 \%$ (9). Further, Neonakis et al. (10) reported a human M. bovis isolate identified as M. bovis subsp. caprae in a Geno-
Type MTBC assay evaluation study.

$M$. caprae, a recently identified separate MTBC species, should be carefully addressed nationally and internationally. Key risk populations in countries not officially TB-free and with sizeable goat populations must be identified, occupational history sought, testing capacity extended, and monitoring and reporting improved. A One Health approach that highlights interdependence of human and animal health sectors is needed to curb the spread of this pathogen.

\section{Acknowledgments}

We thank all members of the staff involved in the investigation of this study at the National Reference Laboratory for Mycobacteria, Sotiria Chest Diseases Hospital, Athens; the National Public Health Organization, Athens; the Aristotle University Pulmonary Department, G. Papanikolaou Hospital, Thessaloniki; the General Directorate of Veterinary Medicine, Athens; and the Directorate of Public Health, Kilkis Prefecture, Kilkis, Greece.

\section{About the Author}

Dr. Papaventsis is a medical director in Clinical Microbiology-Laboratory Medicine at the National Reference Laboratory for Mycobacteria, Sotiria Chest 
Diseases Hospital, Athens, Greece. His research interests include molecular diagnosis and genotyping of tuberculosis and infections caused by nontuberculous mycobacteria.

\section{References}

1. Aranaz A, Cousins D, Mateos A, Domínguez L. Elevation of Mycobacterium tuberculosis subsp. caprae Aranaz et al. 1999 to species rank as Mycobacterium caprae comb. nov., sp. nov. Int J Syst Evol Microbiol. 2003;53:1785-9. https://doi.org/10.1099/ijs.0.02532-0

2. Prodinger WM, Brandstätter A, Naumann L, Pacciarini M, Kubica T, Boschiroli ML, et al. Characterization of Mycobacterium caprae isolates from Europe by mycobacterial interspersed repetitive unit genotyping. J Clin Microbiol. 2005;43:4984-92. https:// doi.org/10.1128/JCM.43.10. 4984-4992.2005

3. Rodríguez S, Bezos J, Romero B, de Juan L, Álvarez J, Castellanos E, et al.; Spanish Network on Surveillance and Monitoring of Animal Tuberculosis. Mycobacterium caprae infection in livestock and wildlife, Spain. Emerg Infect Dis. 2011;17:532-5. https://doi.org/10.3201/eid1703.100618

4. European Food Safety Authority. The European Union One Health 2018 zoonoses report. EFSA J. 2019;17:5926 [cited 2019 Dec 12]. https:/ / www.efsa.europa.eu/en/efsajournal/ pub/5926

5. Kubica T, Rüsch-Gerdes S, Niemann S. Mycobacterium bovis subsp. caprae caused one-third of human M. bovis-associated tuberculosis cases reported in Germany between 1999 and 2001. J Clin Microbiol. 2003;41:3070-7. https:/ / doi.org/ 10.1128/JCM.41.7.3070-3077.2003

6. Allix-Béguec C, Harmsen D, Weniger T, Supply P, Niemann S. Evaluation and strategy for use of MIRUVNTRplus, a multifunctional database for online analysis of genotyping data and phylogenetic identification of Mycobacterium tuberculosis complex isolates. J Clin Microbiol. 2008;46:2692-9. https:// doi.org/10.1128/JCM.00540-08

7. World Organization for Animal Health, World Health Organization, Food and Agricultural Organization of the United Nations. Roadmap for zoonotic tuberculosis. 2017 [cited 2020 Oct 23]. http:// www.oie.int/fileadmin/Home/ eng/Our_scientific_expertise/docs/pdf/Tuberculosis/ Roadmap_zoonotic_TB.pdf

8. Valcheva V, Savova-Lalkovska T, Vyazovaya A, Dimitrova A, Bonovska M, Najdenski H. First insight into phylogeography of Mycobacterium bovis and M. caprae from cattle in Bulgaria. Infect Genet Evol. 2020;81:104240. https://doi.org/10.1016/j.meegid.2020.104240

9. Ikonomopoulos J, Aranaz A, Balaskas C, Sechi L, Gazouli M. Outbreak of acute tuberculosis in a goat herd; first case of Mycobacterium caprae isolation in Greece. Online J Vet Res. 2006;10:108-15

10. Neonakis IK, Gitti Z, Petinaki E, Maraki S, Spandidos DA. Evaluation of the GenoType MTBC assay for differentiating 120 clinical Mycobacterium tuberculosis complex isolates. Eur J Clin Microbiol Infect Dis. 2007;26:151-2. https:/ / doi.org/ 10.1007/s10096-007-0255-y

Address for correspondence: Dimitrios Papaventsis, National Reference Laboratory for Mycobacteria, Sotiria Chest Diseases Hospital, 152 Mesogeion Ave, Athens 11527, Greece; email: papaventsis.d@sotiria.gr,dpapaventsis@gmail.com

\section{Outbreak of Rabbit Hemorrhagic Disease Virus 2 Infection, Ghana}

\author{
Aruna Ambagala, Patrick Ababio, Lindsey Lamboo, \\ Melissa Goolia, Oliver Lung, Yohannes Berhane, \\ Theophilus Odoom
}

Author affiliations: National Centre for Foreign Animal Disease, Canadian Food Inspection Agency, Winnipeg, Manitoba, Canada (A. Ambagala, L. Lamboo, M. Goolia, O. Lung, Y. Berhane); Veterinary Services Directorate, Accra Laboratory, Accra, Ghana (P. Ababio, T. Odoom)

\section{DOI: https://doi.org/10.3201/eid2707.210005}

In September 2019, high mortality in commercial rabbits was reported in the Greater Accra Region of Ghana. Rabbit hemorrhagic disease virus 2 phylogenetically related to isolates from 2015-2017 outbreaks in the Netherlands was confirmed as the causative agent. The virus has not yet been detected in native rabbits in Ghana.

$\mathrm{R}$ abbit hemorrhagic disease (RHD) is an acute, fatal, highly contagious viral hepatitis in European rabbits (Oryctolagus cuniculus) (1). It causes severe economic losses in the rabbit meat and fur industries and can have a substantial negative ecological impact on wild rabbit populations and their predators. The causative agent, RHD virus (RHDV; family Caliciviridae, genus Lagovirus) has a single-stranded positive-sense RNA genome $\approx 7.4 \mathrm{~kb}$ in length. Pathogenic RHDV strains exist in 2 main genotypes, GI.1 and GI.2 (RHDV2). RHDV GI.1 has several variants: GI.1a (proposed as G6/RHDVa), GI.1b (G1), GI.1c (G2), and GI.1d (G3-G5) (2). It is considered enzootic in domestic and wild European rabbits in Asia and Europe; sporadic outbreaks occur in the Americas, Middle East, and Africa (1). RHDV2 GI.2, a variant first reported in France in 2010 (3), differs antigenically from RHDV GI.1 and is therefore considered a distinct serotype. RHDV2, which has replaced RHDV GI.1 in many countries in Europe, infects rabbits of all ages and crosses the species barrier to affect nonEuropean rabbit species (4).

Rabbit production, because of its low costs, has been promoted to reduce poverty in Africa but has been threatened by RHD outbreaks since the late 1980s. In 2015, RHDV2 was detected on Tenerife in the Canary Islands (5). In April 2017, an outbreak was reported in northern Morocco (6) caused by a recombinant GI.1b/GI.1b/GI.2 RHDV2 strain closely related to isolates identified in Portugal in 2014. 